\title{
Post-learning infusion of anisomycin into the anterior cingulate cortex impairs instrumental acquisition through an effect on reinforcer valuation
}

\author{
Sietse Jonkman ${ }^{1}$ and Barry J. Everitt \\ Behavioural and Clinical Neuroscience Institute and Department of Experimental Psychology, University of Cambridge, Cambridge \\ CB2 3EB, United Kingdom
}

\begin{abstract}
The integrity of the rodent anterior cingulate cortex (ACC) is essential for various aspects of instrumental behavior, but it is not clear if the ACC is important for the acquisition of a simple instrumental response. Here, it was demonstrated that post-session infusions of anisomycin into the rat ACC completely prevented the acquisition of instrumental responding. The experimental use of post-session intracranial infusions of plasticity inhibitors is assumed to affect local consolidation of plasticity, but not behavioral task performance. However, in associative appetitive conditioning, postsession intracranial infusion of pharmaco-active compounds could actually interfere with subsequent task performance indirectly through retrospective effects on the valuation of ingested rewards. Thus, it was subsequently demonstrated that the intracranial infusion of anisomycin into the ACC after sucrose pellet consumption significantly reduced subsequent pellet consumption, suggesting that the infusion of anisomycin into the ACC produced conditioned taste avoidance. In the third experiment, an innovative procedure was introduced that dissociated the effects of intracranial infusions after conditioning sessions on task-learning and unconditioned stimulus valuation. With this procedure, the infusion of anisomycin into the ACC after instrumental sessions did not affect instrumental reinforcer valuation or the acquisition of instrumental responding, suggesting that plasticity in the ACC is not necessary for the acquisition of instrumental behavior.
\end{abstract}

The anterior cingulate cortex (ACC) in the rodent brain is the area of association cortex that is most intimately associated with movement control, as it has direct corticospinal projections to motor neurons (Miller 1987) and is intricately connected with motor and premotor cortex (Brecht et al. 2004; Wang et al. 2008), and movement-related discharges in motivated tasks have been observed in the ACC (Jung et al. 1998; Kargo et al. 2007). On the basis of these findings, the ACC has been described as the limbic motor cortex (Craig 2003). Indeed, it has been demonstrated that neural activity in the ACC codes for performed actions and observed appetitive outcomes (Lapish et al. 2008), suggesting that the ACC could be important for instrumental conditioning. However, it is currently unclear whether the role of the ACC in instrumental conditioning is restricted to relatively complex instrumental tasks with response conflict (Lapish et al. 2008) and high response efforts (Rudebeck et al. 2006), or whether it extends generally to the acquisition of a simple appetitively motivated instrumental response as well. For example, it has been reported that the acquisition of instrumental responding under variable ratio 2 schedule of reinforcement was impaired after presession intra-ACC infusions of the $N$-methyl-D-aspartate (NMDA) receptor antagonist AP-5 (McKee et al. 2007).

However, the practical investigation of the neural basis of learning is complicated by what can be considered as "side effects" of manipulations that are intended to interfere selectively with learning mechanisms. Both pretraining lesions and presession infusions of pharmacological agents into specific brain regions can produce interference with basic task performance in addition to impairing task-related learning. This problem can be overcome by

'Corresponding author.

E-mail sj306@cam.ac.uk; fax 44-01223-333564.

Article is online at http://www.learnmem.org/cgi/doi/10.1101//m1497709. the use of infusions of pharmacological agents after a behavioral conditioning session, specifically to target the consolidation of learning. Positive effects of post-session intracranial infusions of protein synthesis inhibitors such as anisomycin (Rosenblum et al. 1993), or NMDA, and dopamine receptor antagonists such as AP-5 or SCH 23390 (Dalley et al. 2005) on the acquisition of conditioned responses can be interpreted more confidently in terms of interference with learning, at least when combined with negative effects in delayed-infusion control groups. Certainly, positive effects of post-session infusions on learning in the absence of effects of delayed control infusions cannot be attributed to interference with task performance.

Nevertheless, post-session intracranial infusions may still cause confounding effects on conditioned behavior in appetitive conditioning experiments, by changing the valuation of the unconditioned stimulus (US), or reward, in subsequent sessions. Such manipulations could reduce the valuation of rewards either through interference with consolidation or reconsolidation of the sensory or motivational properties of the US (Wang et al. 2005; Pedroza-Llinás et al. 2009), which would lead to persistent neophobia, or through retrospective devaluation of the task reward through the post-ingestion induction of an aversive state. Neither effect would be expected to be apparent in delayed-infusion control conditions. Furthermore, considering that animals typically earn far fewer rewards in conditioning sessions than they would maximally consume if rewards were freely available, such effects would not necessarily be visible in terms of consumption of earned rewards and unaffected latencies to collect rewards.

In view of these considerations, the effect of the infusion of anisomycin into the rat ACC after instrumental sessions on the acquisition of conditioned responding was investigated. Based on the obtained results, it was further investigated whether the infusion of anisomycin into the ACC after free consumption of 
a novel reward would affect subsequent pellet consumption. Finally, a third experiment was conducted that introduced an innovative procedure that was designed to dissociate the effects of intracranial infusion of protein synthesis inhibitors after conditioning sessions on task-learning and US valuation.

\section{Results}

\section{Experiment 1: Instrumental acquisition}

This experiment investigated the effects of infusion of anisomycin into the ACC after free-operant instrumental conditioning sessions on the subsequent acquisition and consolidation of instrumental responding. Anisomycin was infused after each of the first five instrumental acquisition sessions, and there were three conditions: immediate phosphate buffered saline (PBS) infusions, immediate anisomycin infusions, and 6-h delayed anisomycin infusions.

The effects of anisomycin infusions into the ACC on the number of active lever presses are illustrated in Figure 1A. In the overall three-way analysis, there was a significant effect of time $\left(F_{(2,22)}=88.9, P<0.001\right)$, a significant effect of treatment $\left(F_{(2,22)}=\right.$ $9.8, P=0.001)$, and a significant interaction between these factors

\section{A}

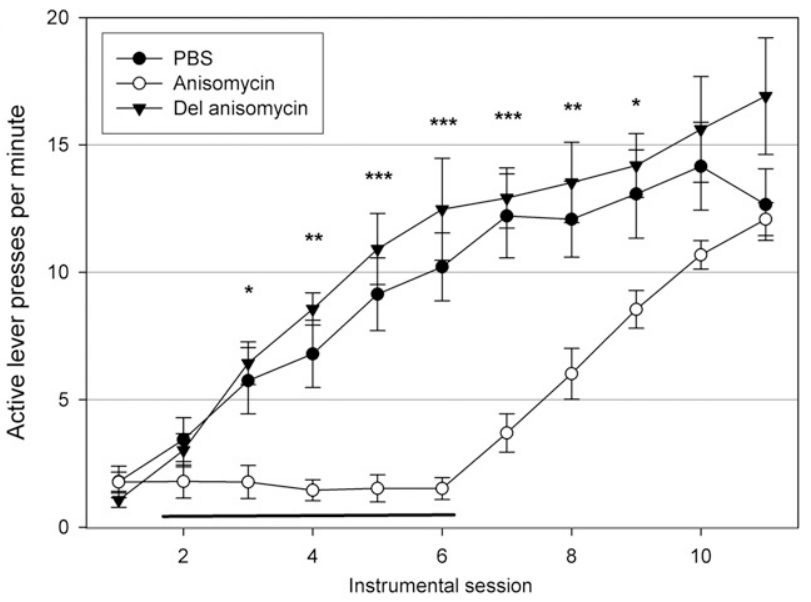

B

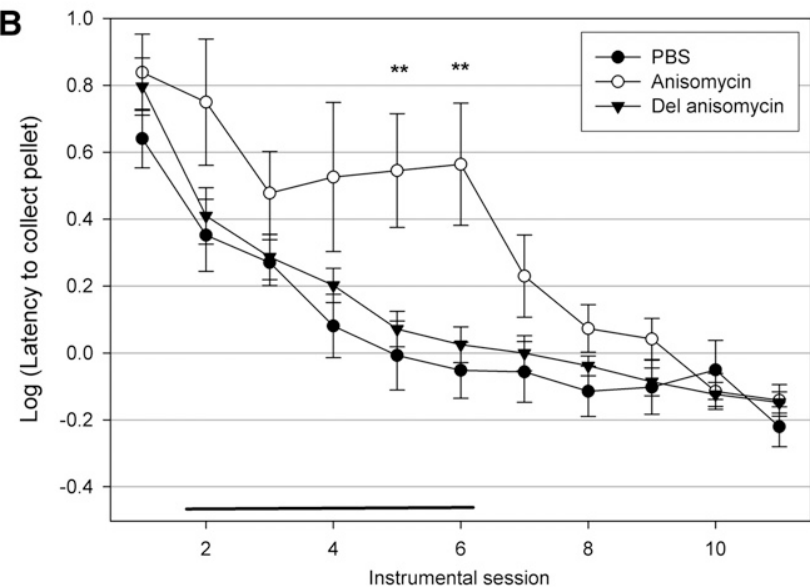

Figure 1. Post-session infusions of anisomycin into the ACC significantly affected the acquisition of instrumental responding. The mean ( \pm SEM) active lever presses per minute $(A)$ and log of the latency to collect earned pellets $(B)$ were both significantly affected by anisomycin infusions. PBS, anisomycin, or delayed anisomycin (6 h) was infused after the first five sessions, the effects of which were measured in sessions 2-6, as denoted by the black bars. Asterisks denote a significant difference between PBS and anisomycin groups: ${ }^{*} P<0.05,{ }^{* *} P<0.01,{ }^{* *} P<0.001$.
$\left(F_{(2,22)}=6.2, P<0.001\right)$. A separate two-way ANOVA of the PBS and delayed anisomycin groups showed that there was a significant effect of time $\left(F_{(1,16)}=65.1, P<0.001\right)$, but no effect of treatment $\left(F_{(1,16)}<1, P=\mathrm{NS}\right)$ or interaction $\left(F_{(1,16)}=1.4, P=\mathrm{NS}\right)$. On the other hand, analysis of the PBS and anisomycin groups showed that there was a significant effect of time $\left(F_{(1,15)}=62.3, P<0.001\right)$, a significant effect of treatment $\left(F_{(1,15)}=12.5, P=0.003\right)$, and a significant interaction between these factors $\left(F_{(1,15)}=9.7, P<\right.$ $0.001)$. A Sidak post-hoc analysis revealed that the anisomycintreated group pressed the active lever significantly less in sessions $3(P=0.020), 4(P=0.003), 5(P<0.001), 6(P<0.001), 7(P<0.001)$, $8(P=0.005)$, and $9(P=0.038)$. Thus, immediate post-session anisomycin infusions, but not delayed anisomycin infusions, completely prevented the acquisition of instrumental responding.

Comparable effects were found in the analysis of earned reinforcers. There was a significant effect of time $\left(F_{(2,22)}=98.2, P<\right.$ $0.001)$, a significant effect of treatment $\left(F_{(2,22)}=17.3, P>0.001\right)$, and a significant interaction $\left(F_{(2,22)}=7.5, P<0.001\right)$. A separate two-way ANOVA of the PBS and delayed anisomycin groups showed that there was a significant effect of time $\left(F_{(1,16)}=96.7\right.$, $P<0.001)$, but no effect of treatment $\left(F_{(1,16)}<1, P=\mathrm{NS}\right)$ or interaction $\left(F_{(1,16)}=1.1, P=\mathrm{NS}\right)$. Analysis of the PBS and anisomycin groups showed that there was a significant effect of time $\left(F_{(1,15)}=55.7, P<0.001\right)$, a significant main effect of treatment $\left(F_{(1,15)}=16.5, P<0.001\right)$, and a significant interaction $\left(F_{(1,15)}=8.0, P<0.001\right)$. A Sidak post-hoc analysis revealed that the anisomycin-treated group earned fewer reinforcers in sessions 4 $(P=0.003), 5(P<0.001), 6(P<0.001), 7$ ( $P<0.001), 8$ ( $P=0.004), 9$ $(P=0.009)$, and $11(P=0.038)$.

The number of inactive lever presses was not affected by time $\left(F_{(2,22)}=1.3, P=\mathrm{NS}\right)$, or treatment $\left(F_{(2,22)}=1.6, P=\mathrm{NS}\right)$, but there was a significant interaction between these factors $\left(F_{(2,22)}=2.2, P=\right.$ 0.023). A comparison of the PBS and delayed anisomycin groups showed that there was a significant effect of time $\left(F_{(1,16)}=2.7, P=\right.$ $0.004)$, but no effect of treatment $\left(F_{(1,16)}=1.4, P=\mathrm{NS}\right)$ or interaction $\left(F_{(1,16)}<1, P=\mathrm{NS}\right)$. Analysis of the PBS and anisomycin groups showed that there was no effect of time $\left(F_{(1,15)}=1.1, P=\right.$ NS) and no effect of treatment $\left(F_{(1,15)}<1, P=\mathrm{NS}\right)$, but there was a significant interaction between these factors $\left(F_{(1,15)}=2.8, P=\right.$ $0.023)$. A Sidak post-hoc analysis revealed that the anisomycintreated group pressed the inactive lever less only in session $4(P=$ 0.003).

The number of magazine entries per session was not affected by time $\left(F_{(2,22)}=2.8, P=0.029\right)$, but it was significantly affected by treatment $\left(F_{(2,22)}=7.9, P=0.003\right)$ and by the interaction of these factors $\left(F_{(2,22)}=6.7, P<0.001\right)$. A two-way ANOVA of the PBS and delayed anisomycin groups showed that there was no effect of time $\left(F_{(1,16)}=1.4, P=\mathrm{NS}\right)$ and that there was a significant effect of treatment $\left(F_{(1,16)}=8.3, P=0.012\right)$, but no interaction $\left(F_{(1,16)}=2.3\right.$, $P=$ NS). Thus, the rats in the delayed anisomycin group entered the magazine more often than did the rats in the PBS group. Analysis of the PBS and anisomycin groups showed that there was a significant effect of time $\left(F_{(1,15)}=8.0, P=0.001\right)$ and no significant main effect of treatment $\left(F_{(1,15)}<1, P=\mathrm{NS}\right)$, but there was a significant interaction between these factors $\left(F_{(1,15)}=\right.$ 9.8, $P<0.001$ ). A Sidak post-hoc analysis revealed that the anisomycin-treated group entered the magazine less often in sessions $3(P=0.004), 4(P<0.001), 5(P<0.001)$, and $6(P=$ $0.001)$, but more often in sessions $9(P=0.024), 10(P=0.001)$, and $11(P<0.001)$.

The log of the latency to collect the pellet after delivery was significantly affected by time $\left(F_{(2,22)}=56.0, P<0.001\right)$, demonstrating that the rats learned to collect the pellets with decreasing latencies throughout the experiment, by treatment $\left(F_{(2,22)}=4.4\right.$, $P=0.026)$, and the interaction of these factors $\left(F_{(2,22)}=3.0, P=\right.$ 0.003). A separate two-way ANOVA of the PBS and delayed 
anisomycin groups showed that there was a significant effect of time $\left(F_{(1,16)}=72.5, P<0.001\right)$, but no effect of treatment $\left(F_{(1,16)}<1\right.$, $P=\mathrm{NS})$ or interaction $\left(F_{(1,16)}=1.1, P=\mathrm{NS}\right)$. Analysis of the PBS and anisomycin groups showed that there was a significant effect of time $\left(F_{(1,15)}=29.6, P<0.001\right)$, a significant main effect of treatment $\left(F_{(1,15)}=5.9, P=0.029\right)$, and a significant interaction between these two factors $\left(F_{(1,15)}=4.0, P=0.007\right)$. A Sidak post-hoc analysis revealed that the anisomycin-treated group was significantly slower to pick up earned reinforcers in sessions $5(P=0.009)$ and $6(P<0.005)$, as illustrated in Figure $1 \mathrm{~B}$. All rats ate all earned pellets by the end of the sessions.

Taken together, these results show that the post-session infusions of anisomycin into the ACC impaired instrumental acquisition as reflected in both active lever presses and earned reinforcers. In fact, the infusion of anisomycin into the ACC completely prevented the increase in lever presses and earned reinforcers between sessions that was observed in control conditions, and when infusions were discontinued, both active lever presses and earned reinforcers started to increase gradually in a manner that was indistinguishable from normal early acquisition. This effect was specific to the immediate post-session infusion of anisomycin, as anisomycin infusions that were delayed by $6 \mathrm{~h}$ did not affect any measure of instrumental conditioning. The infusion of anisomycin into the ACC also decreased the number of inactive lever presses and magazine entries, but reward collection latencies and reward consumption were not substantially affected. These results suggest that instrumental acquisition is mediated by plasticity in the ACC.

\section{Experiment 2: Sucrose pellet valuation}

The second experiment examined the effects of infusion of anisomycin into the ACC after free consumption of sucrose pellets on subsequent pellet consumption. There were six free-consumption sessions per subject: one baseline test; one short-term test $1 \mathrm{~h}$ later; three long-term tests on days 3, 6, and 9 (the first two of which were also followed by intra-ACC infusions); and a final recovery test on day 10 . There were three conditions: immediate PBS infusions, immediate anisomycin infusions, and $6 \mathrm{~h}$ delayed anisomycin infusions.

An overall three-way ANOVA of pellet consumption showed that there was a significant effect of time $\left(F_{(1,16)}=15.9, P<0.001\right)$, a significant effect of treatment $\left(F_{(1,16)}=19.1, P<0.001\right)$, and a significant interaction between these factors $\left(F_{(1,16)}=12.5, P<\right.$ 0.001). A two-way ANOVA comparing pellet consumption of PBS and delayed anisomycin-infused rats showed that there was a significant effect of time $\left(F_{(1,11)}=43.7, P<0.001\right)$, reflecting loss of neophobia, but there was no effect of treatment $\left(F_{(1,11)}=1.3, P=\right.$ NS) or significant interaction between these factors $\left(F_{(1,11)}=1, P=\right.$ NS). A two-way ANOVA comparing the pellet consumption of PBSand anisomycin-infused rats showed that there was no significant effect of time $\left(F_{(1,11)}=6.9, P=0.051\right)$, there was a significant effect of treatment $\left(F_{(1,11)}=18.8, P=0.001\right)$, and a significant interaction between these factors $\left(F_{(1,11)}=12.7, P<0.001\right)$.

A Sidak post-hoc analysis showed that pellet consumption was not significantly different in the preinfusion baseline test $(P=$ 0.203). However, $1 \mathrm{~h}$ after infusion, the consumption of pellets in the second test was significantly lower in the anisomycin-infused group $(P=0.001)$. The consumption of pellets was also significantly lower in the anisomycin-infused rats in the three long-term effect tests on days $3(P=0.009), 6(P=0.001)$, and $9(P=0.001)$, all tested $3 \mathrm{~d}$ after a post-ingestion infusion. Finally, when infusions were discontinued, there was no recovery of pellet consumption in the anisomycin-treated group by day 10, as evident by significantly lower pellet consumption in the anisomycin group $(P=0.002)$.

These results are illustrated in Figure 2 and demonstrate that the infusion of anisomycin into the ACC immediately after

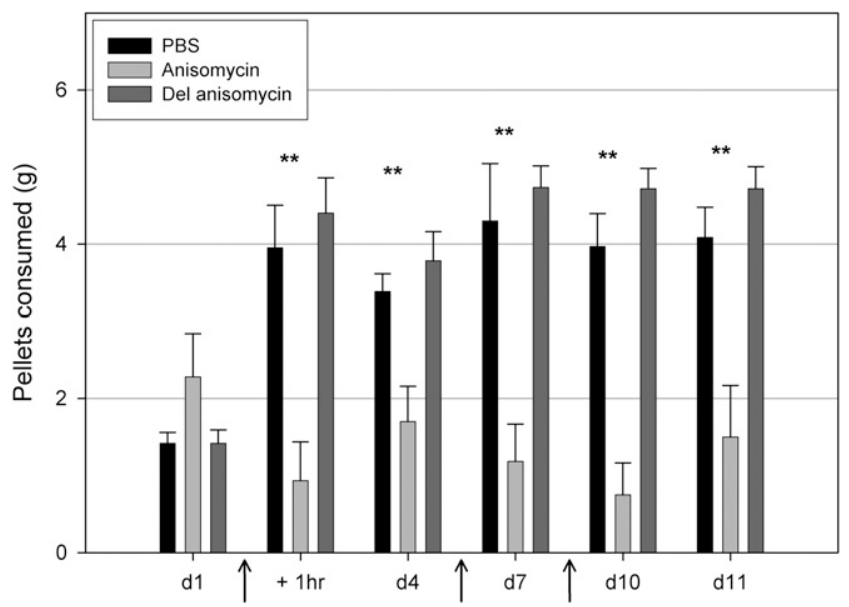

Figure 2. Anisomycin infusions into the ACC after consumption of pellets produced a decrease in subsequent pellet consumption that was evident both at short- and long-term tests, and did not recover when infusions were discontinued. Arrows below the $x$-axis denote intracranial infusions. Asterisks denote a significant difference between PBS and anisomycin groups: ${ }^{* *} P<0.01$.

consumption of pellets produced conditioned taste avoidance. The infusion of anisomycin into the ACC greatly reduced pellet consumption at both short and longer intervals, suggesting that this effect was not mediated by the prevention of consolidation of US value or US identity learning. Furthermore, the effect of intraACC infusions on pellet consumption persisted when infusions were discontinued. These effects were not due to the proactive effects of anisomycin, as they were absent in the delayed infusion control group.

\section{Experiment 3: Instrumental acquisition with US protection}

The third experiment reinvestigated the effects of post-session infusion of anisomycin into the ACC on instrumental acquisition, controlling for potential US valuation effects through the introduction of a brief consumption session of an alternative reinforcer (lemon-flavored maltodextrin solution) after instrumental sessions with sucrose pellet reinforcement and before infusions. Compared with the instrumental sucrose pellet reward, maltodextrin was consumed in closer temporal proximity to the aversive state-inducing infusion, and unlike sucrose pellets, maltodextrin was a completely novel ingestive reward for the rats at the time of the first intra-ACC infusion. Such conditions are expected to lead to the attribution of the post-consumption aversive state to the more recently consumed maltodextrin, rather then sucrose pellet consumption (Kalat 1974). Anisomycin was infused after each of the first five instrumental sessions, and there were two conditions: immediate PBS infusions and immediate anisomycin infusions.

The effects of anisomycin infusions on maltodextrin consumption were as anticipated. An ANOVA of the maltodextrin consumption after the first five instrumental sessions showed that there was no effect of time $\left(F_{(1,17)}=2.0, P=\mathrm{NS}\right)$, but there was a significant main effect of treatment $\left(F_{(1,17)}=99.2, P<0.001\right)$ and a significant interaction between these factors $\left(F_{(1,17)}=14.9, P<\right.$ 0.001). A Sidak post-hoc analysis showed that the anisomycininfused rats consumed significantly less in the second $(P<0.001)$, third $(P<0.001)$ fourth $(P<0.001)$, and fifth $(P<0.001)$ freeconsumption sessions. However, there was no significant difference in sucrose pellet consumption in the 15-min free-feeding session inside the operant chamber $2 \mathrm{~d}$ after the fifth instrumental 
session and infusion $\left(F_{(1,17)}<1, P=\mathrm{NS}\right)$. These results confirm that the instrumental US valuation was protected successfully and are illustrated in Figure 3.

The effects of anisomycin infusions into the ACC on the acquisition of instrumental responding in the modified procedure are illustrated in Figure 4A. ANOVA of the active lever presses showed that there was a significant effect of time $\left(F_{(1,17)}=34.8, P<\right.$ $0.001)$, reflecting successful instrumental learning, but there was no effect of treatment $\left(F_{(1,17)}<1, P=\mathrm{NS}\right)$ or interaction $\left(F_{(1,17)}=\right.$ $1.5, P=$ NS). Similarly, the number of earned reinforcers was significantly modulated by time $\left(F_{(1,17)}=48.0, P<0.001\right)$, but not by treatment $\left(F_{(1,17)}<1, P=\mathrm{NS}\right)$, and there was no interaction between these factors $\left(F_{(1,17)}<1, P=\mathrm{NS}\right)$. The number of inactive lever presses was not affected by time $\left(F_{(1,17)}=1.1, P=\mathrm{NS}\right)$, by treatment $\left(F_{(1,17)}=2.5, P=\mathrm{NS}\right)$, or by the interaction of these factors $\left(F_{(1,17)}<1, P=\mathrm{NS}\right)$. The number of magazine entries was not significantly modulated by time $\left(F_{(1,17)}=1.2, P=\mathrm{NS}\right)$, treatment $\left(F_{(1,17)}<1, P=\mathrm{NS}\right)$, or their interaction $\left(F_{(1,17)}<1, P=\right.$ NS). Finally, the log of the latency to enter the magazine after a reward was delivered was significantly influenced by time $\left(F_{(1,17)}=\right.$ 9.5, $P<0.001)$, but there was no main effect of treatment on this measure $\left(F_{(1,17)}=1.1, P=\mathrm{NS}\right)$ and no interaction between these factors $\left(F_{(1,17)}<1, P=\mathrm{NS}\right)$, as illustrated in Figure $4 \mathrm{~B}$. All rats ate all pellets by the end of the sessions.

Thus, the infusion of anisomycin into the ACC after instrumental conditioning sessions did not affect any measure of instrumental acquisition when the effects of anisomycin on US valuation were controlled for.

\section{Discussion}

The results of these experiments demonstrate that post-session anisomycin infusions into the ACC impaired the acquisition of instrumental responding. However, when infused into the ACC after pellet consumption, anisomycin reduced subsequent pellet consumption at both short and long delays, paradoxically suggesting that anisomycin produced aversion learning, where the valuation of an ingestive reward is decreased by post-ingestion induction of some form of discomfort, illness, or distress. Thus, the infusion of anisomycin into the ACC most likely induced an aversive state. However, when the effects of the aversive properties

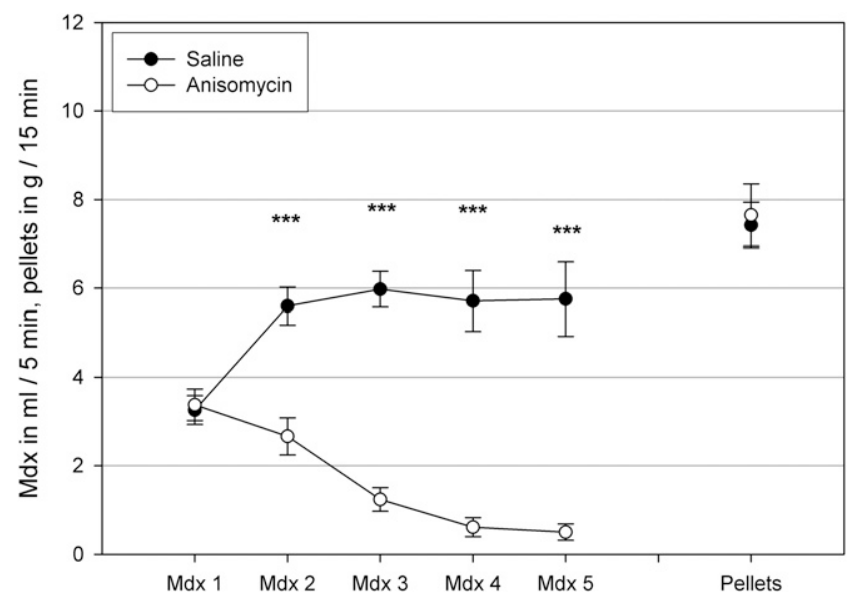

Figure 3. Interposition of maltodextrin consumption between instrumental sessions and anisomycin infusions into the ACC preserved pellet valuation and induced a significant aversion to maltodextrin. Data are presented as mean ( \pm SEM) milliliters of maltodextrin consumption per 5 -min session or as grams of pellet consumption per 15-min session in the operant chamber. Asterisks denote a significant difference between PBS and anisomycin groups: ${ }^{* *} P<0.001$.
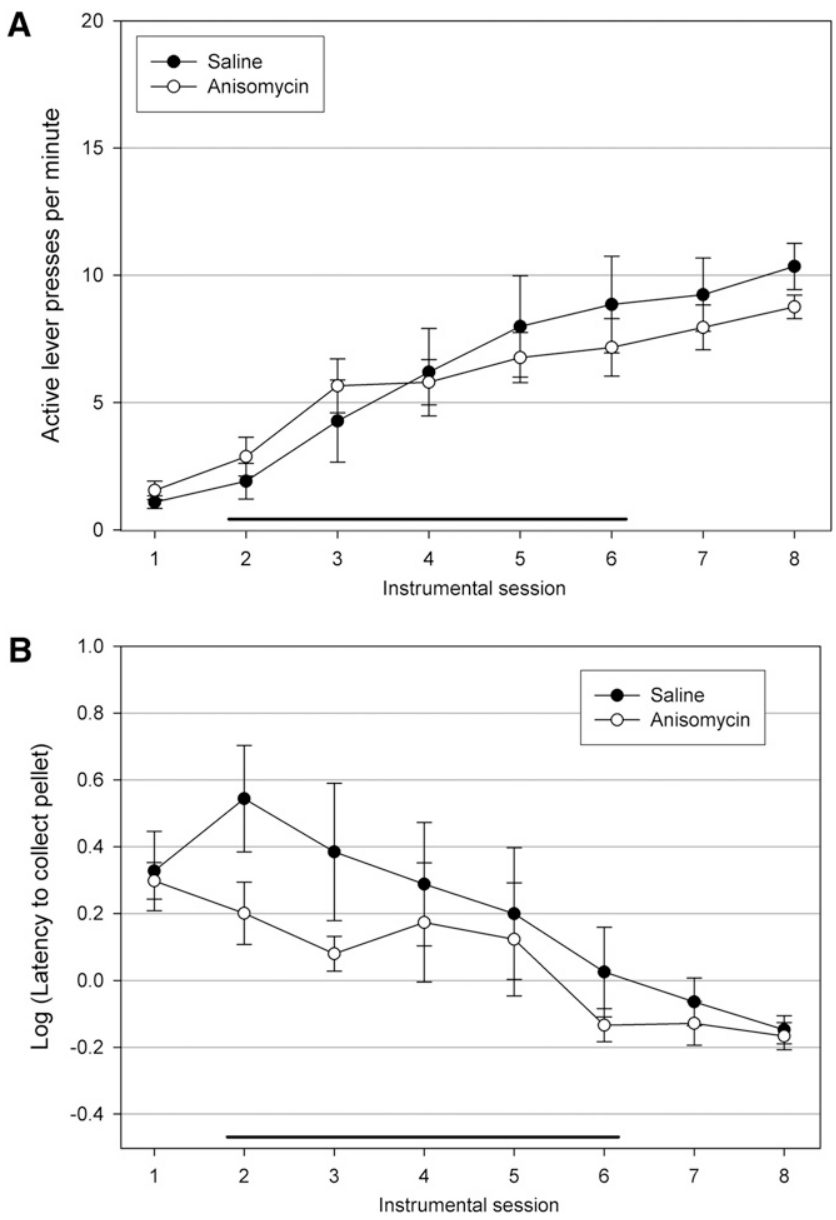

Figure 4. Post-session infusions of anisomycin into the ACC did not affect the acquisition of instrumental responding when effects on pellet valuation were prevented. The mean ( \pm SEM) active lever presses per minute $(A)$ and log of the latency to collect earned pellets $(B)$ were both unaffected by anisomycin infusions. PBS or anisomycin was infused after the first five sessions, the effects of which were measured in sessions 2-6.

of intra-ACC anisomycin infusions on sucrose pellet valuation were neutralized in a novel procedure, instrumental acquisition was not impaired by post-session intra-ACC infusions of anisomycin, indicating that this medial prefrontal area does not contribute to instrumental conditioning.

The present results suggest that plasticity in the ACC is not necessary for the acquisition of an instrumental response. This result is not consistent with a report of impaired acquisition of instrumental responding under a variable ratio 2 schedule of reinforcement after presession intra-ACC infusions of the NMDA receptor antagonist AP-5 (McKee et al. 2007). The discrepancy between these two reports could have been due to interference with some aspect of task performance or US valuation in the AP-5 study, or to the difference in schedule employed (ratio vs. interval). However, early in training, ratio and interval schedules of reinforcement are very similar (Dickinson 1985) and so the difference in the schedule of reinforcement is perhaps not a very likely explanation for the discrepancy. The present finding that plasticity in the ACC is not necessary for the acquisition of instrumental responding is consistent with the finding that pretraining lesions of the ACC did not affect the acquisition of responding under ascending interval schedules of reinforcement (Cardinal et al. 2003). Furthermore, pretraining lesions of the 
entire neocortex only moderately impaired instrumental acquisition under fixed interval 40-sec conditions (Jaldow et al. 1990).

The aversive effect of intracranial anisomycin infusions is a novel finding that has major implications for the use of this agent in the study of consolidation mechanisms underlying appetitive and aversive behavior. It has been reported that systemic injections of the protein synthesis inhibitor anisomycin ( 5 and $10 \mathrm{mg} / \mathrm{kg}$ ) after pellet consumption led to a consequent taste avoidance (Hernandez and Kelley 2004). In addition, this conditioned taste avoidance produced a significant reduction in instrumental responses that was not due to direct interference with task performance. However, two other studies reported no effects of systemic post-ingestion anisomycin injections of even larger doses (30 and $150 \mathrm{mg} / \mathrm{kg}$ ) on subsequent water or sucrose solution consumption, respectively (Patterson et al. 1987; Blaiss and Janak 2007). It was not clear from these experiments whether the observed reduction in sucrose consumption after post-consumption intra-ACC anisomycin infusions was due to taste aversion or mere taste avoidance (Parker 1995); but given that intra-ACC infusions of glutamate have been reported to produce a helplesslike state (Hunter et al. 2003), it is possible that intra-ACC anisomycin infusions may have resulted in taste avoidance by inducing a stressful state.

Although used to probe neural plasticity mechanisms, it has not previously been investigated whether intracranial infusion of anisomycin after reward consumption produces conditioned taste avoidance. These results suggest that positive effects of postsession intracranial infusions of anisomycin on appetitive learning that includes consumption of ingestive rewards without controlling for alterations in US value cannot be interpreted exclusively in terms of memory formation. Indeed, there are several such reports in the literature that may need to be reinvestigated and confirmed in further studies (Hernandez et al. 2002; De la Cruz et al. 2008).

The aversive effect of anisomycin in the ACC was probably not mediated by the prevention of consolidation of local plasticity, as it was already fully apparent $1 \mathrm{~h}$ after consumption. It is more likely that the aversive state was produced by some activating property of anisomycin, presumably changing local cellular excitability. In principle, the known activating effect of anisomycin on the intracellular mitogen activated protein kinases c-Jun N-terminal kinase (JNK) and p38 (Stadheim and Kucera 2002) could have fulfilled this role. However, the aversive effect could also be caused by the marked monoamine efflux that has been reported after anisomycin administration into the basolateral amygdala (Canal et al. 2007), although anisomycin-induced monoamine efflux has not been investigated directly in the ACC. If anisomycin infusion into the ACC produced acute monoamine efflux, neural excitability would have altered quickly and dramatically.

However, another question concerns how conditioned taste avoidance could result from altered neuronal excitability in a brain region where anisomycin is preventing plasticity. It would have to be assumed that anisomycin either did not prevent local plasticity or ultimately activated another brain region in which the conditioned taste avoidance was stored. It is unlikely that anisomycin did not prevent taste avoidance-related plasticity in the ACC for two reasons. First, intracranial anisomycin is a potent amnesic agent that reliably impairs memory consolidation and reconsolidation in a wide variety of learning paradigms, including conditioned taste aversion after infusion into the gustatory cortex (Rosenblum et al. 1993; Abel et al. 1997; Schafe and LeDoux 2000; Morris et al. 2006; Power et al. 2006; Touzani et al. 2007). Second, the ACC has not previously been implicated in taste avoidance learning. It has been reported that the ACC is involved in the expression of remote ( $1 \mathrm{mo})$, but not recently learned $(1 \mathrm{~d})$, conditioned taste avoidance (Ding et al. 2008). However, the conditioned taste avoidance expressed in the present experiments was clearly recently learned $(1 \mathrm{~h})$. Therefore, the conditioned taste avoidance was most likely caused by activation of aversive signal projections into primary taste aversion learning areas, such as the insular gustatory cortex (Rosenblum et al. 1993), the central amygdala (Lamprecht et al. 1997), or the nucleus accumbens (Fenu et al. 2001) which were presumably activated by the taste of either novel sucrose or maltodextrin. The valuation of novel ingestive rewards may be particularly susceptible to such effects, as the aversion signal need not be specific and the consumption of a novel taste presumably increases the sensitivity of gustatory brain regions to potentially aversive signals.

Indeed, there is clear evidence that local activity in the ACC can produce an aversive signal that is incorporated into aversive memories that are stored in other brain regions. Thus, local application of the glutamatergic receptor agonist homocysteic acid induced an aversive signal that produced conditioned place avoidance, the memory of which was not itself stored in the ACC (Johansen and Fields 2004). The aversive signals produced by intra-ACC homocysteic acid and anisomycin infusions may therefore share a glutamatergic mechanistic basis.

In conclusion, the present results suggest that plasticity in the ACC is not necessary for the acquisition of instrumental responding. Furthermore, these results suggest that the use of post-session infusions of putative plasticity inhibitors in the study of appetitive conditioning can produce disruptive latent effects on reinforcer valuation, and positive effects of such infusions on appetitive learning should be interpreted with caution. A novel procedure has been demonstrated that can dissociate the effects of such infusions on US valuation and instrumental response acquisition, allowing for a secure interpretation of behavioral results.

\section{Materials and Methods}

\section{Subjects}

Male outbred Lister hooded rats (Charles River, Kent, United Kingdom), weighing 270-350 g at the start of the experiments were housed in pairs in polycarbonate cages (length, $40 \mathrm{~cm}$; width, $25 \mathrm{~cm}$; height, $18 \mathrm{~cm}$ ) and maintained under a reversed 12-h light/ dark cycle (lights on at 7:00 p.m.) at a constant temperature $\left(21^{\circ} \mathrm{C} \pm 1^{\circ} \mathrm{C}\right)$ with free access to water. Each subject was given $18 \mathrm{~g}$ of laboratory chow (SDS Ltd.) per day, which was sufficient to maintain body weight at no less than $85 \%$ of free-feeding weight. The experimental procedures were conducted in accordance with the United Kingdom's 1986 Animals (Scientific Procedures) Act (project license PPL 80/17672 + 280/2234).

\section{Apparatus}

Instrumental training and testing took place in six operant conditioning chambers $(29.5 \times 32.5 \times 23.5 \mathrm{~cm}$; Med Associates $)$ equipped with two 4-cm-wide retractable levers that were mounted in the intelligence panel $12 \mathrm{~cm}$ apart and $8 \mathrm{~cm}$ from the grid floor. A white house light $(2.5 \mathrm{~W}, 24 \mathrm{~V})$ was located on the opposite wall. A pellet dispenser delivered individual $45 \mathrm{mg}$ food pellets, (Noyes Formula P pellets; Sandown Scientific) into a recessed magazine $(3.8 \times 5.5 \mathrm{~cm})$ situated between the levers. Entry into the magazine was detected by the interruption of an infrared source. The floor of the chamber was covered with a metal grid with bars separated by $1 \mathrm{~cm}$. The testing chamber was placed within a soundand light-attenuating housing equipped with a ventilation fan that also screened external noise. The operant conditioning chambers were controlled by software written in $\mathrm{C}++$ using the Whisker control system (http://www.whiskercontrol.com). Free consumption of pellets or lemon-flavored maltodextrin solution $(3 \% \mathrm{w} / \mathrm{v}$ lemon juice, Sainsbury's Lemon Juice, $20 \% \mathrm{w} / \mathrm{v}$ maltodextrin, Myprotein.co.uk) was conducted in separate polycarbonate cages (length, $40 \mathrm{~cm}$; width, $25 \mathrm{~cm}$; height, $18 \mathrm{~cm}$ ). 


\section{Stereotaxic surgery}

Subjects were anaesthetized with ketamine (Ketalar, $90 \mathrm{mg} / \mathrm{kg}$ i.p.) and xylazine (Rompun, $6.7 \mathrm{mg} / \mathrm{kg}$, i.p.) and placed in a stereotaxic frame (David Kopf Instruments) with the incisor bar set at $-3.3 \mathrm{~mm}$ below the interaural line (Paxinos and Watson 1998). Bilateral 22 gauge, stainless-steel guide cannula (Coopers Needleworks) were implanted $1.5 \mathrm{~mm}$ above the ACC (coordinates from bregma: anterior-posterior, $+1.4 \mathrm{~mm}$; medial-lateral, $\pm 0.9 \mathrm{~mm}$; dorsal-ventral from skull, $-1.7 \mathrm{~mm}$ ), using standard stereotaxic techniques and secured to the skull using bone screws and dental cement. Twenty-nine-gauge obturators flush with the end of the guide cannula were inserted in the guide cannula. After surgery, rats were allowed a recovery period of at least $7 \mathrm{~d}$ before behavioral testing.

\section{Drug infusions}

Before the start of experimental infusions, the rats were habituated to the infusion procedure with two surrogate infusions, which consisted of the removal and replacement of the obturator during gentle restraint within a time course identical to that of drug infusion. During infusions, the rats were gently restrained while the obturators were removed and a 29-gauge bilateral injector that protruded $1.5 \mathrm{~mm}$ beyond the guide cannula was inserted, and $0.5 \mu \mathrm{L}$ was then infused over a 1-min period into each hemisphere. The injector was left in place for $1 \mathrm{~min}$ to allow the drug to diffuse in the local vicinity of the injector tip. The injector was then carefully removed, and the obturator was replaced. All infusions were given in the behavioral testing room. Anisomycin (Sigma) was dissolved in an equimolar concentration of $\mathrm{HCl}$, adjusted to $\mathrm{pH} 7.2$, and brought to a concentration of $125 \mu \mathrm{g} / \mu \mathrm{L}$ in PBS, resulting in an effective dose of $62.5 \mu \mathrm{g} / \mathrm{side}$. This dose of anisomycin was selected on the basis of previous findings (Rosenblum et al. 1993), showing $>90 \%$ inhibition of protein synthesis in the cortex for $2 \mathrm{~h}$.

\section{Behavioral procedures}

\section{Experiment 1}

The purpose of this experiment was to investigate whether intraACC infusions of anisomycin after instrumental sessions affected instrumental acquisition. Instrumental acquisition was studied under conditions that have been described and characterized quantitatively previously (Jonkman et al. 2009). There were three conditions: immediate PBS infusions $(N=9)$, immediate anisomycin infusions $(N=7)$, and $6 \mathrm{~h}$ delayed anisomycin infusions $(N=8)$.

\section{Magazine training}

All animals received two 15-min sessions of magazine training in the operant chamber with the lever retracted on days 1 and 2 . During each of these sessions, pellets were delivered under a variable time (VT) 30-sec (range, 1-59 sec) schedule.

\section{Instrumental acquisition}

Beginning on day 3, all rats received five 15-min instrumental training sessions with five post-session drug infusions. After infusions, rats were left undisturbed for the next $2 \mathrm{~d}$ to allow washout of the drugs, resulting in instrumental sessions and infusions on days $3,6,9,12$, and 15 . All instrumental sessions operated a fixed interval 30-sec schedule of reinforcement (FI 30sec) and started with the insertion of two levers and the onset of the house light. Either the left or right lever operated the instrumental contingency, while pressing the other lever had no programmed consequences. Designation of active and inactive levers was counterbalanced across conditions.

\section{Recovery}

After the cessation of infusions, the rats were given six more daily FI 30-sec sessions on days 16, 17, 18, 19, 20, and 21 to allow recovery of performance of experimental groups to control levels in order to demonstrate that the observed effects were not due to tissue damage in the ACC.
The number of active lever presses and earned pellets were the primary measures of instrumental acquisition, and the number of inactive lever presses and magazine entries were recorded as measures of locomotor activity. The latency to enter the magazine after reward delivery was recorded as a measure of reward collection learning. The log of this measure was taken for analysis due to large outliers.

\section{Experiment 2}

The purpose of this experiment was to investigate whether intraACC infusions of anisomycin after sucrose pellet consumption affected subsequent pellet consumption. There were three conditions: immediate PBS infusions $(N=6)$, immediate anisomycin infusions $(N=6)$, and $6 \mathrm{~h}$ delayed anisomycin infusions $(N=6)$.

\section{Baseline}

On day 1 , all rats were individually placed in a plastic prefeeding chamber containing a full bowl of pellets and were allowed to consume this novel reward for $5 \mathrm{~min}$. An intracranial infusion was given immediately after consumption.

\section{Short-term effect}

The short-term effect of infusion on subsequent pellet consumption was measured $1 \mathrm{~h}$ after infusion, by a second 5 -min freefeeding session in the prefeeding chamber with the pellet reward.

\section{Long-term effect}

The long-term effect of infusion on pellet consumption was measured $3 \mathrm{~d}$ after the first infusion on day 4 by a third 5-min free-feeding session that was followed by a second intracranial infusion. The rats were left undisturbed for $72 \mathrm{~h}$ to allow drug washout and prevent proactive effects of anisomycin, which have been observed up to $24 \mathrm{~h}$, but not $48 \mathrm{~h}$ after intracerebral infusion (Canal et al. 2007). The second long-term test was conducted $3 \mathrm{~d}$ after the first on day 7, also involved a 5-min free-feeding session, and was followed by an intracranial infusion. The effect of this infusion was tested $3 \mathrm{~d}$ later on day 10 in the fifth 5-min freefeeding test, which was not followed by an infusion.

\section{Recovery}

In order to investigate whether a potential effect of intracranial infusion on US valuation was transient, a final 5-min freeconsumption recovery test was conducted $1 \mathrm{~d}$ after the last longterm effect test on day 11.

\section{Experiment 3}

The purpose of this experiment was to investigate whether intraACC infusions of anisomycin after instrumental sessions affected instrumental acquisition when US valuation was kept constant. There were two conditions: immediate PBS infusions $(N=9)$ and immediate anisomycin infusions $(N=9)$.

\section{Pellet exposure}

All rats were placed in the operant chamber for 15 min with the house light on and 30 pellets pre-placed in the magazine on days $1-3$.

\section{Magazine training}

All animals received two 15-min sessions of magazine training in the operant chamber with the lever retracted on days 4 and 5 . During each of these sessions, pellets were delivered under a VT 30 -sec (range, 1-59 sec) schedule.

\section{Instrumental acquisition}

Beginning on day 6 , all rats received five 15 -min instrumental training sessions with five post-session drug infusions on days 6,9 , 12,15 , and 18. All instrumental training sessions operated on a FI 30 -sec schedule of reinforcement and started with the insertion of two levers and the onset of the house light. Either the left or right lever operated the instrumental contingency, while pressing the 

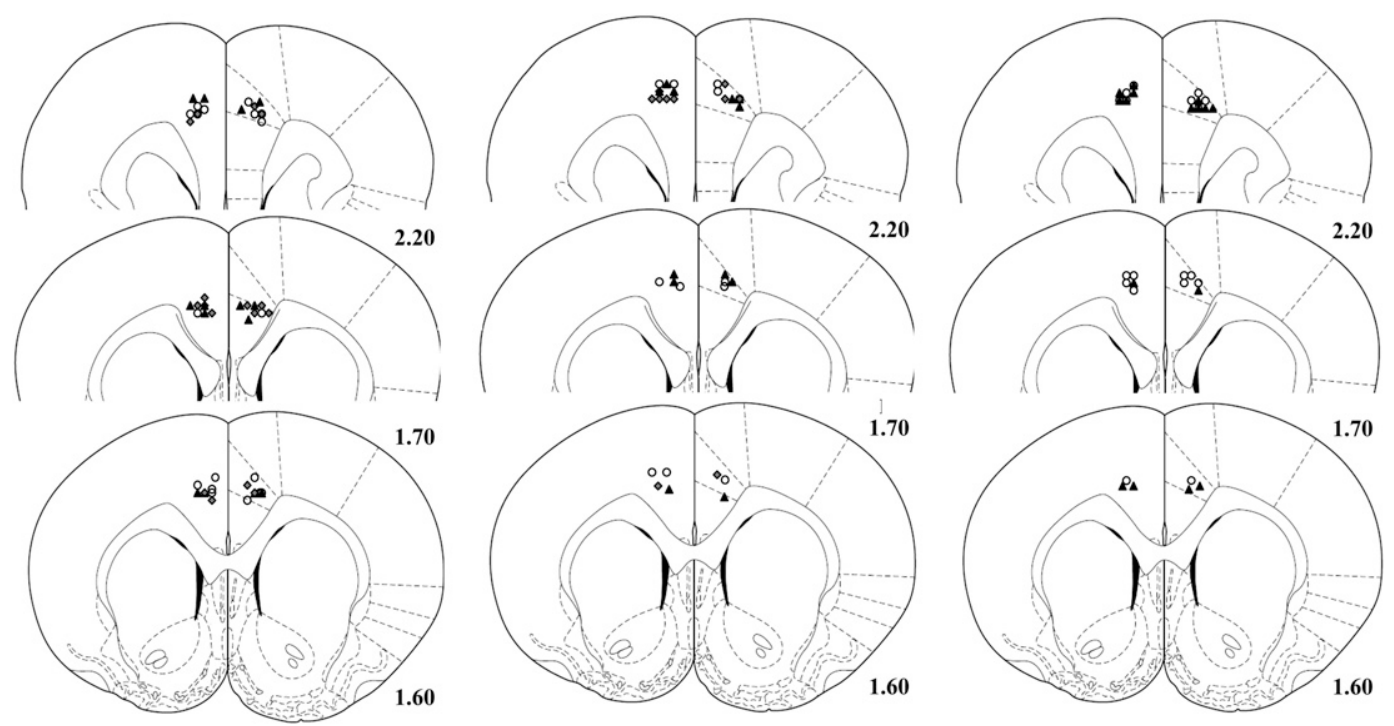

Figure 5. Neuroanatomical representation of cannula placements in experiments $1(A), 2(B)$, and $3(C)$. Open circles represent PBS-treated rats, solid black triangles represent anisomycin-treated rats, and gray diamonds represent delayed anisomycin-treated rats. Sections at various distances from bregma in millimeters.

other lever had no programmed consequences. Designation of active and inactive levers was counterbalanced across conditions. Immediately after each of the five instrumental sessions, the rats were placed in the plastic free-feeding chambers for $5 \mathrm{~min}$ and allowed to drink lemon-flavored maltodextrin solution $(3 \%[\mathrm{w} / \mathrm{v}]$ lemon juice, $20 \%[\mathrm{w} / \mathrm{v}]$ maltodextrin). Maltodextrin consumption was immediately followed by intracranial infusion of anisomycin. After completion of the fifth cycle, the rats were allowed to freely consume pellets for $15 \mathrm{~min}$ from a filled bowl inside the operant chamber on day 21, in order to confirm that pellet valuation was successfully normalized.

\section{Recovery}

After cessation of infusions, the rats were given three more daily FI 30 -sec sessions on days 22,23 , and 24 to allow recovery of instrumental performance.

\section{Histology}

Following the completion of the experiment, subjects were anaesthetized with a lethal dose of sodium pentobarbitone (Euthatal, $200 \mathrm{mg} / \mathrm{mL}$; Genus Express) and perfused transcardially with 0.01 M PBS followed by $4 \%$ paraformaldehyde. The brains were removed and post-fixed in paraformaldehyde. Prior to being sectioned, the brains were transferred to $20 \%$ sucrose in $0.2 \mathrm{M}$ phosphate buffer and left overnight. Coronal sections were cut at $60 \mu \mathrm{m}$ on a freezing microtome and stained with cresyl violet. Cannula locations were mapped onto standardized sections of the rat brain (Paxinos and Watson 1998). Only rats with infusion cannula tip placements were included in the data analysis. For the included rats, there was no evidence for gross damage to the cortex beyond the location of the guide and infusion cannula. The neuroanatomical representation of the cannula placements in the three experiments is illustrated in Figure 5.

\section{Statistics}

Statistical analysis was performed using ANOVAs with appropriate between- and within-subject factors. Significant interactions were analyzed at separate time points with Sidak post-hoc tests (Howell 1997). All statistical tests used an $\alpha$ level of $P<0.05$ for the rejection of the null hypothesis.

\section{Acknowledgments}

This work was supported by United Kingdom Medical Research Council (MRC) grant 9536855 and was conducted within the
MRC/Wellcome Trust Behavioural and Clinical Neuroscience Institute.

\section{References}

Abel T, Nguyen PV, Barad M, Deuel TAS, Kandel ER, Bourtchouladze R. 1997. Genetic demonstration of a role for PKA in the late phase of LTP and in hippocampus-based long-term memory. Cell 88: 615-626.

Blaiss CA, Janak PH. 2007. Post-training, but not post-reactivation, administration of amphetamine and anisomycin modulates Pavlovian conditioned approach. Neurobiol Learn Mem 87: 644-658.

Brecht M, Krauss A, Muhammad S, Sinai-Esfahani L, Bellanca S, Margrie TW. 2004. Organization of rat vibrissa motor cortex and adjacent areas according to cytoarchitectonics, microstimulation, and intracellular stimulation of identified cells. J Comp Neurol 479: 360-373.

Canal CE, Chang Q, Gold PE. 2007. Amnesia produced by altered release of neurotransmitters after intra-amygdala injections of a protein synthesis inhibitor. Proc Natl Acad Sci 104: 12500-12505.

Cardinal RN, Parkinson JA, Marbini HD, Toner AJ, Bussey TJ, Robbins TW, Everitt BJ. 2003. Role of the anterior cingulate cortex in the control over behavior by Pavlovian conditioned stimuli in rats. Behav Neurosci 117: 566-587.

Craig AD. 2003. Interoception: The sense of the physiological condition of the body. Curr Opin Neurobiol 13: 500-505.

Dalley JW, Laane K, Theobald DEH, Armstrong HC, Corlett PR, Chudasama Y, Robbins TW. 2005. Time-limited modulation of appetitive Pavlovian memory by D1 and NMDA receptors in the nucleus accumbens. Proc Natl Acad Sci 102: 6189-6194.

De la Cruz V, Rodriguez-Ortiz CJ, Balderas I, Bermudez-Rattoni F. 2008. Medial temporal lobe structures participate differentially in consolidation of safe and aversive taste memories. Eur J Neurosci 28: 1377-1381.

Dickinson A. 1985. Actions and habits: The development of behavioural autonomy. Philos Trans R Soc Lond B Biol Sci 308: 67-78.

Ding HK, Teixeira Ct M, Frankland PW. 2008. Inactivation of the anterior cingulate cortex blocks expression of remote, but not recent, conditioned taste aversion memory. Learn Mem 15: 290293.

Fenu S, Bassareo V, Di Chiara G. 2001. A role for dopamine D1 receptors of the nucleus accumbens shell in conditioned taste aversion learning. J Neurosci 21: 6897-6904.

Hernandez PJ, Kelley AE. 2004. Long-term memory for instrumental responses does not undergo protein synthesis-dependent reconsolidation upon retrieval. Learn Mem 11: 748-754.

Hernandez PJ, Sadeghian K, Kelley AE. 2002. Early consolidation of instrumental learning requires protein synthesis in the nucleus accumbens. Nat Neurosci 5: 1327-1331.

Howell DC. 1997. Statistical methods for psychology, 4th ed. Duxbury Press, Belmont, CA. 
Hunter AM, Balleine BW, Minor TR. 2003. Helplessness and escape performance: Glutamate-adenosine interactions in the frontal cortex. Behav Neurosci 117: 123-135.

Jaldow EJ, Oakley DA, Davey GC. 1990. Performance on two fixed-interval schedules in the absence of neocortex in rats. Behav Neurosci 104: 763777.

Johansen JP, Fields HL. 2004. Glutamatergic activation of anterior cingulate cortex produces an aversive teaching signal. Nat Neurosci 7: 398-403.

Jonkman S, Mar AC, Dickinson A, Robbins TW, Everitt BJ. 2009. The rat prelimbic cortex mediates inhibitory response control but not the consolidation of instrumental learning. Behav Neurosci 123: 875885 .

Jung MW, Qin Y, McNaughton BL, Barnes CA. 1998. Firing characteristics of deep layer neurons in prefrontal cortex in rats performing spatial working memory tasks. Cereb Cortex 8: 437-450.

Kalat JW. 1974. Taste salience depends on novelty, not concentration, in taste-aversion learning in the rat. J Comp Physiol Psychol 86: 47-50

Kargo WJ, Szatmary B, Nitz DA. 2007. Adaptation of prefrontal cortical firing patterns and their fidelity to changes in action-reward contingencies. J Neurosci 27: 3548-3559.

Lamprecht R, Hazvi S, Dudai Y. 1997. cAMP response element-binding protein in the amygdala is required for long- but not short-term conditioned taste aversion memory. J Neurosci 17: 8443-8450.

Lapish CC, Durstewitz D, Chandler LJ, Seamans JK. 2008. Successful choice behavior is associated with distinct and coherent network states in anterior cingulate cortex. Proc Natl Acad Sci 105: 11963-11968.

McKee BL, Andrzejewski ME, Dyvig HR, Baldo BA, Kelley AE. 2007. $\mathrm{N}$-methyl-D-aspartate (NMDA) receptor blockade in the anterio cingulate cortex impairs acquisition of instrumental responding for sucrose. In Proceedings of the Society for Neuroscience Meeting. Society for Neuroscience, San Diego, CA.

Miller MW. 1987. The origin of corticospinal projection neurons in rat. Exp Brain Res 67: 339-351.

Morris RGM, Inglis J, Ainge JA, Olverman HJ, Tulloch J, Dudai Y, Kelly PAT. 2006. Memory reconsolidation: Sensitivity of spatial memory to inhibition of protein synthesis in dorsal hippocampus during encoding and retrieval. Neuron 50: 479-489.
Parker LA. 1995. Rewarding drugs produce taste avoidance, but not taste aversion. Neurosci Biobehav Rev 19: 143-151.

Patterson TA, Rosenzweig MR, Bennett EL. 1987. Amnesia produced by anisomycin in an appetitive task is not due to conditioned aversion. Behav Neural Biol 47: 17-26.

Paxinos G, Watson C. 1998. The rat brain in stereotaxic coordinates, 4 th ed. Academic, New York.

Pedroza-Llinás R, Ramírez-Lugo L, Guzmán-Ramos K, Zavala-Vega S, Bermúdez-Rattoni F. 2009. Safe taste memory consolidation is disrupted by a protein synthesis inhibitor in the nucleus accumbens shell. Neurobiol Learn Mem 92: 45-52.

Power AE, Berlau DJ, McGaugh JL, Steward O. 2006. Anisomycin infused into the hippocampus fails to block "reconsolidation" but impairs extinction: The role of re-exposure duration. Learn Mem 13: 27-34

Rosenblum K, Meiri N, Dudai Y. 1993. Taste memory: The role of protein synthesis in gustatory cortex. Behav Neural Biol 59: 49-56.

Rudebeck PH, Walton ME, Smyth AN, Bannerman DM, Rushworth MFS 2006. Separate neural pathways process different decision costs. Nat Neurosci 9: 1161-1168.

Schafe GE, LeDoux JE. 2000. Memory consolidation of auditory Pavlovian fear conditioning requires protein synthesis and protein kinase $\mathrm{A}$ in the amygdala. J Neurosci 20: RC96:1-5.

Stadheim TA, Kucera GL. 2002. c-Jun N-terminal kinase/stress-activated protein kinase (JNK/SAPK) is required for mitoxantrone- and anisomycin-induced apoptosis in HL-60 cells. Leuk Res 26: 55-65.

Touzani K, Puthanveettil SV, Kandel ER. 2007. Consolidation of learning strategies during spatial working memory task requires protein synthesis in the prefrontal cortex. Proc Natl Acad Sci 104: 5632-5637.

Wang S-H, Ostlund SB, Nader K, Balleine BW. 2005. Consolidation and reconsolidation of incentive learning in the amygdala. J Neurosci 25: 830-835.

Wang Y, Matsuzaka Y, Mushiake H, Shima K. 2008. Spatial distribution of cingulate cortical cells projecting to the primary motor cortex in the rat. Neurosci Res 60: 406-411.

Received June 4, 2009; accepted in revised form September 11, 2009. 


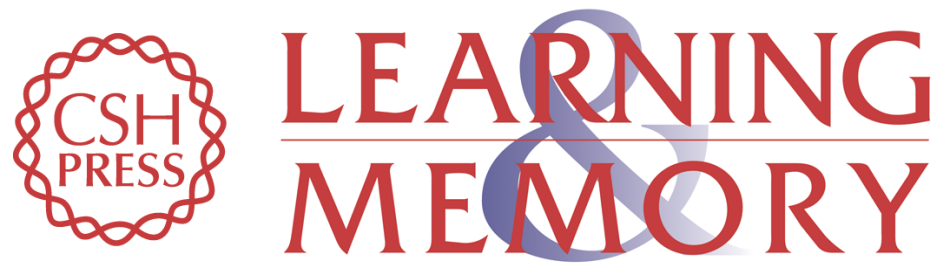

\section{Post-learning infusion of anisomycin into the anterior cingulate cortex impairs instrumental acquisition through an effect on reinforcer valuation}

Sietse Jonkman and Barry J. Everitt

Learn. Mem. 2009, 16:

Access the most recent version at doi:10.1101//m.1497709

References This article cites 35 articles, 11 of which can be accessed free at: http://learnmem.cshlp.org/content/16/11/706.full.html\#ref-list-1

License

Email Alerting

Receive free email alerts when new articles cite this article - sign up in the box at the Service top right corner of the article or click here. 